\title{
A NECESSARY CONDITION FOR PRINCIPAL CLUSTER SETS TO BE VOID
}

\author{
C. L. BELNA ${ }^{1}$
}

Abstract. Let $f$ be an arbitrary function from the open unit disk $D$ into the Riemann sphere $W$, and let $p$ be a point on the unit circle $C$. We prove that if the principal cluster set of $f$ at $p$ is void, then either $p$ is an ambiguous point of $f$ or the diameter of each arc-cluster set of $f$ at $p$ is greater than a fixed positive number.

An arc $\sigma \subset D$ is an arc at $p$ if $\sigma \cup\{p\}$ is a continuous image of the closed interval $[0,1]$. For each $\operatorname{arc} \sigma$ at $p$, the set of all points $w \in W$ for which there exists a sequence $\left\{z_{k}\right\} \subset \sigma$ with $z_{k} \rightarrow p$ and $f\left(z_{k}\right) \rightarrow w$ is denoted by $C(f, p, \sigma)$, and its diameter (relative to the chordal metric) is denoted by $|C(f, p, \sigma)|$. A point $p \in C$ is an ambiguous point of $f$ if there exist two arcs $\sigma_{1}$ and $\sigma_{2}$ at $p$ for which

$$
C\left(f, p, \sigma_{1}\right) \cap C\left(f, p, \sigma_{2}\right)=\varnothing .
$$

The principal cluster set of $f$ at $p$ is the set

$$
\Pi(f, p)=\cap C(f, p, \sigma)
$$

where the intersection is taken over all $\operatorname{arcs} \sigma$ at $p$.

THEOREM. Let $f$ be an arbitrary function from $D$ into $W$ and let $p$ be a point on $C$. If $\Pi(f, p)=\varnothing$, then either (1) $p$ is an ambiguous point of $f$ or (2) there exists a positive number $h$ such that $|C(f, p, \sigma)| \geqq h$ for each arc $\sigma$ at $p$.

Proof. Suppose that condition (2) does not hold. Then, there exists a sequence $\left\{\sigma_{k}\right\}$ of arcs at $p$ with $\left|C\left(f, p, \sigma_{k}\right)\right| \rightarrow 0$. By choosing a subsequence of $\left\{\sigma_{k}\right\}$ if necessary, we may assume that there exists a point $w^{*} \in W$ for which

$$
\left|C\left(f, p, \sigma_{k}\right) \cup\left\{w^{*}\right\}\right| \rightarrow 0 .
$$

Choose an $\operatorname{arc} \sigma$ at $p$. If $p$ is not an ambiguous point of $f$, there exists a point

$$
w_{k} \in C(f, p, \sigma) \cap C\left(f, p, \sigma_{k}\right)
$$

Received by the editors May 15, 1969.

AMS Subject Classification. Primary 3062.

Key Phrases. Principal cluster set, arc-cluster set diameter, ambiguous point.

1 This result is contained in the author's doctoral thesis written under the direction of Professor Peter Lappan at Michigan State University. 
for each positive integer $k$. Since $C(f, p, \sigma)$ is closed and $w_{k} \rightarrow w^{*}$, it follows that $w^{*} \in C(f, p, \sigma)$. Then, since $\sigma$ represents an arbitrary arc at $p, w^{*} \in \Pi(f, p)$ in violation of $\Pi(f, p)=\varnothing$. Hence $p$ is an ambiguous point of $f$ and the theorem is proved.

REMARK. In case $f$ is holomorphic in $D$, this theorem is an immediate consequence of Theorem 1 in McMillan's paper [1].

\section{REFERENCE}

1. J. E. McMillan, Curvilinear oscillations of holomorphic functions, Duke Math. J. 33 (1966), 495-498. MR 34 \#1527.

Michigan State University and

Wright State University 